\title{
A Nearly-optimal Fano-based Coding Algorithm
}

\author{
Luis G. Rueda* and B. John Oommen ${ }^{\dagger}$
}

\begin{abstract}
Statistical coding techniques have been used for a long time in lossless data compression, using methods such as Huffman's algorithm, arithmetic coding, Shannon's method, Fano's method, etc. Most of these methods can be implemented either statically or adaptively. In this paper, we show that although Fano coding is suboptimal, it is possible to generate static Fano-based encoding schemes which are arbitrarily close to the optimal, i.e. those generated by Huffman's algorithm. By taking advantage of the properties of the encoding schemes generated by this method, and the concept of "code word arrangement", we present an enhanced version of the static Fano's method, namely Fano ${ }^{+}$. We formally analyze Fano ${ }^{+}$by presenting some properties of the Fano tree, and the theory of list rearrangements. Our enhanced algorithm achieves compression ratios arbitrarily close to those of Huffman's algorithm on files of the Calgary corpus and the Canterbury corpus.
\end{abstract}

\section{Introduction}

\subsection{Problem Statement}

Huffman's algorithm is a well-known encoding method that generates an optimal prefix encoding scheme, in the sense that the average code word length is minimum. As opposed to this, Fano's method has not been used so much because it generates prefix encoding schemes that can be sub-optimal.

In this paper, we present an enhancement of the traditional Fano's method by which encoding schemes, which are arbitrarily close to the optimum, can be easily constructed.

\subsection{Overview}

We assume that we are given a source alphabet, $\mathcal{S}=\left\{s_{i}, \ldots, s_{m}\right\}$, whose probabilities of occurrence are $\mathcal{P}=$ $\left[p_{1}, \ldots, p_{m}\right]$, and a code alphabet, $\mathcal{A}=\left\{a_{1}, \ldots, a_{r}\right\}$. We intend to generate an encoding scheme, $\left\{s_{i} \rightarrow w_{i}\right\}$, in such a way that $\bar{\ell}=\sum_{i=1}^{m} p_{i} \ell_{i}$ is minimized, where $\ell_{i}$ is the length of $w_{i}$. Although, we specifically consider the binary code alphabet, the generalization to the $r$-ary case is not too intricate.

Lossless encoding methods used to solve this problem include Huffman's algorithm (Huffman, 1952), Shannon's method (Shannon and Weaver, 1949), arithmetic coding (Sayood, 2000), Fano's method (Hankerson

\footnotetext{
*Member, IEEE. School of Computer Science, University of Windsor, 401 Sunset Ave., Windsor, ON, N9B 3P4, Canada. Email: lrueda@uwindsor.ca. Partially supported by Departamento de Informática, Universidad Nacional de San Juan, Argentina, and NSERC, the Natural Science and Engineering Research Council of Canada. A preliminary version of this paper was presented at the 2001 IEEE Conference on Systems, Man and Cybernetics, Tucson, Arizon, USA.

${ }^{\dagger}$ Fellow, IEEE. School of Computer Science, Carleton University, 1125 Colonel By Dr., Ottawa, ON, K1S 5B6, Canada. E-mail: oommen@scs.carleton.ca. Partially supported by NSERC, the Natural Science and Engineering Research Council of Canada.
} 
et al., 1998), etc. Adaptive versions of these methods have been proposed, and can be found in (Faller, 1973; Gallager, 1978; Hankerson et al., 1998; Knuth, 1985; Rueda, 2002; Sayood, 2000). Our survey is necessarily brief as this is a well-reputed field.

We assume that the source is memoryless or zeroth-order, which means that the occurrence of the next symbol is independent of any other symbol that has occurred previously. Higher-order models include Markov models (Hankerson et al., 1998), dictionary techniques (Ziv and Lempel, 1977; Ziv and Lempel, 1978), prediction with partial matching (Witten et al., 1999), grammar based compression (Kieffer and Yang, 2000), etc., and the techniques introduced here are also readily applicable for such "structure" models.

\subsection{Our Contribution}

Huffman's algorithm proceeds by generating the so called Huffman tree, by recursively merging symbols (nodes) into a new conceptual symbol which constitutes an internal node of the tree. In this way, Huffman's algorithm generates the tree in a bottom-up fashion. As opposed to this, Fano's method proceeds by generating a coding tree as well, but it proceeds in a top-down fashion. At each step, the list of symbols is partitioned into two (or more, if the output alphabet is non-binary) new sublists, generating two or more new nodes in the corresponding coding tree. Although Fano's method, typically, generates a sub-optimal encoding scheme ${ }^{1}$, the loss in compression ratio with respect to Huffman's algorithm can be relatively small, but can also be quite significant if the optimality of the partitioning is small.

The binary alphabet version of Fano's method proceeds by partitioning the list of symbols into two new sublists in such a way that the sums of probabilities of these two new sublists are as close to being equal as possible. This procedure is recursively applied to the new sublists until two atomic sublists with a single symbol are obtained, and simultaneously a bit is appended to the code words of each symbol in these sublists. As a result of this, if we start with the probabilities of occurrence in a decreasing order, it can be seen that, occasionally, symbols with higher probabilities are assigned to longer code words than those with lower probabilities. This condition is not desirable; we attempt to rectify this condition in Fano ${ }^{+}$, a superior Fanobased scheme which we develop in this paper.

On the other hand, after constructing a coding tree (such as a Huffman tree or a Fano tree), an encoding scheme is generated from that tree by labeling the branches with the code alphabet (typically, binary) symbols. Given a tree constructed from a source alphabet of $m$ symbols, $2^{m-1}$ different encoding schemes can be generated. Of these, only one of them is of a family of codes known as canonical codes, in which the code words are arranged in a lexicographical order (Witten et al., 1999). Canonical codes are desired because they allow extremely fast decoding, and require approximately half of the space used by a decoding tree. These codes are generated by Fano coding in a natural way.

In this paper, we introduce Fano ${ }^{+}$, an enhanced version of the static Fano coding approach utilizing the concept which we called "code word arrangement", and which is based on a fundamental property of two lists arranged in an increasing and decreasing order respectively (Hardy et al., 1959). In our context, these lists are the code words (in terms of their lengths) and their probabilities respectively. This paper formally details the encoding scheme generation algorithm, the partitioning procedures suitable for $\mathrm{Fano}^{+}$, and a rigorous analysis

\footnotetext{
${ }^{1}$ Even if optimal partition is in $\mathbf{P}$, Fano coding is still sub-optimal (Storer, 1988).
} 
of their respective properties.

We finally discuss some empirical results obtained from running the static Huffman's algorithm, the static version of the traditional Fano coding, and $\mathrm{Fano}^{+}$, on real life data. Our empirical results show that the compression ratios achieved by Fano ${ }^{+}$are comparable to those of other optimal encoding methods such as Huffman's algorithm. Although we use the zeroth order statistical model, other structure/statistical models such as higher-order models, dictionary models, etc., can also be used in conjunction with Fano ${ }^{+}$to achieve compression ratios that are close to those attained by most well known compression schemes.

\section{Properties of the Traditional Fano Coding}

Consider the source alphabet $\mathcal{S}=\left\{s_{1}, \ldots, s_{m}\right\}$ with probabilities of occurrence $\mathcal{P}=\left[p_{1}, \ldots, p_{m}\right]$, where $p_{1} \geq p_{2} \geq \ldots \geq p_{m}$. Unless otherwise stated, in this paper, we assume that the code alphabet is $\mathcal{A}=\{0,1\}$.

We define an encoding scheme as a mapping, $\phi: s_{1} \rightarrow w_{1}, \ldots, s_{m} \rightarrow w_{m}$, where $w_{i} \in \mathcal{A}^{+}$, for $i=1, \ldots, m$. One of the properties of the encoding schemes generated by Huffman's algorithm is that $\ell_{1} \leq \ell_{2} \leq \ldots \leq \ell_{m}$, where $\ell_{i}$ is the length of $w_{i}$. In general, this property is not satisfied by the encoding schemes generated by Fano's method. We also introduce a definition that is important to our work.

Definition 1. Let $\mathcal{S}=\left\{s_{1}, \ldots, s_{m}\right\}$ be the source alphabet whose probabilities of occurrence are $\mathcal{P}=$ $\left[p_{1}, \ldots, p_{m}\right]$, and let $\mathcal{A}=\{0,1\}$ be the code alphabet. A binary coding tree, $\mathcal{T}=\left\{t_{1}, \ldots, t_{2 m-1}\right\}$, is a binary tree in which:

(i) Every node has zero or two children.

(ii) Every internal node, $t_{k}$, has a weight, $\tau_{k}$, calculated as the sum of the weights of its two children.

(iii) Every leaf, $t_{j}$, has a symbol, $s_{i}$, associated with it, and a weight, $\tau_{j}$, which represents the probability of occurrence of $s_{i}$, namely $p_{i}$.

It is also important to mention that if the code word lengths are not in this order, the binary coding tree does not satisfy a fundamental property called the sibling property (Gallager, 1978; Hankerson et al., 1998), which is defined as follows.

Definition 2. Let $\mathcal{T}=\left\{t_{1}, \ldots, t_{2 m-1}\right\}$ be a binary coding tree, whose weights are $\left\{\tau_{1}, \ldots, \tau_{2 m-1}\right\}$, and where $t_{1}$ is the root node. $\mathcal{T}$ satisfies the sibling property if the following conditions hold:

(i) $\tau_{2} \geq \ldots \geq \tau_{2 m-1}$

(ii) $t_{2 k}$ and $t_{2 k+1}$ are siblings in $\mathcal{T}$, for $k=1, \ldots, m-1$.

Before we proceed with the theoretical analysis of the relation between the code word lengths and the sibling property, we recursively define the length of the path in a binary coding tree.

Definition 3. Consider a binary coding tree $\mathcal{T}=\left\{t_{1}, \ldots, t_{2 m-1}\right\}$ in which each leaf is associated with a source alphabet symbol, $s_{i}$. The code word length of a node $t_{j}$, where $t_{j}$ is any node of the tree, is defined as follows:

(i) 1 if $t_{j}$ is a leaf, or 


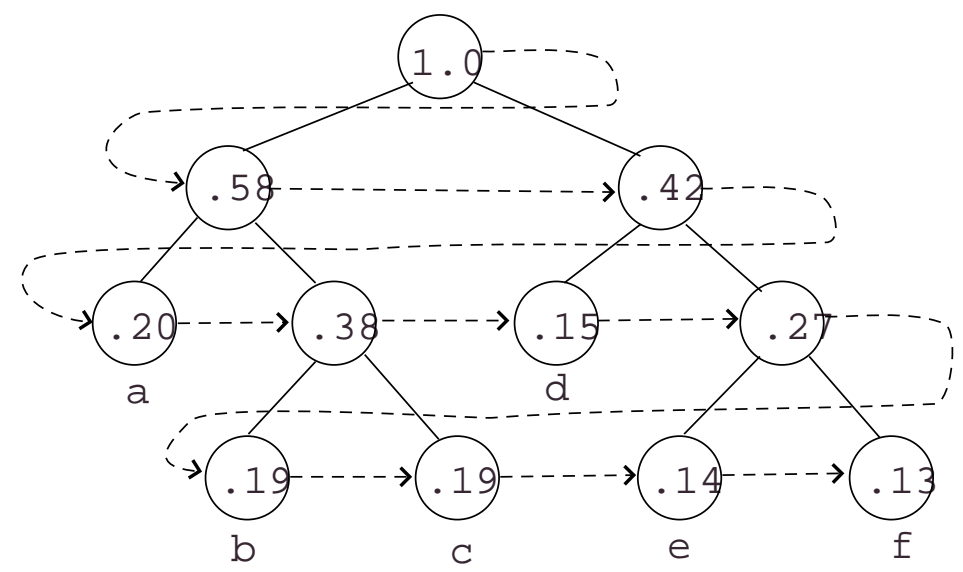

Figure 1: An example of a binary coding tree, which was constructed by following the principles of Fano coding, and does not satisfy the sibling property.

(ii) $1+$ length $\left(t_{j_{c}}\right)$, where $t_{j_{c}}$ is the left or right child of $t_{j}$, whenever $t_{j}$ is an internal node.

Using this definition, we shall prove that whenever the probabilities of occurrences of the input symbols are in a non-increasing order, if the code words are in a non-decreasing order of length, the binary coding tree associated with such an encoding scheme does not satisfy the sibling property. This fact is clarified in Example 1 below and formally stated and proved in Theorem 1. It is thereafter used in Fano ${ }^{+}$to modify the binary coding tree so as to get an enhanced performance.

Example 1. Consider the source alphabet, $\mathcal{S}=\{a, b, c, d, e, f\}$, and the probabilities of occurrence $\mathcal{P}=$ $[.2, .19, .19, .15, .14, .13]$. Suppose that a particular binary coding tree, $\mathcal{T}$, constructed from $\mathcal{S}$ and $\mathcal{P}$ following the principles of Fano coding is the one depicted in Figure 1.

Note that the list positions that we referred to as $t_{i}$, and the positions of the symbols of $\mathcal{S}$ in the list are $t_{k_{i}}$, which are listed in the following tables:

\begin{tabular}{|c|c|}
\hline List Id & $\tau_{i}$ \\
\hline \hline$t_{1}$ & 1.00 \\
\hline$t_{2}$ & .58 \\
\hline$t_{3}$ & .42 \\
\hline$t_{4}$ & .20 \\
\hline$t_{5}$ & .38 \\
\hline$t_{6}$ & .15 \\
\hline$t_{7}$ & .27 \\
\hline$t_{8}$ & .19 \\
\hline$t_{9}$ & .19 \\
\hline$t_{10}$ & .14 \\
\hline$t_{11}$ & .13 \\
\hline
\end{tabular}

\begin{tabular}{|l|r|}
\hline$t_{k_{i}}$ & $k_{i}$ \\
\hline \hline$t_{k_{1}}$ & 4 \\
\hline$t_{k_{2}}$ & 8 \\
\hline$t_{k_{3}}$ & 9 \\
\hline$t_{k_{4}}$ & 6 \\
\hline$t_{k_{5}}$ & 10 \\
\hline$t_{k_{6}}$ & 11 \\
\hline
\end{tabular}

The complete table of the indices, the probabilities of occurrence, $\mathcal{P}$, and the code word lengths, $\left\{\ell_{i}\right\}$, for the symbols are given in the following table: 


\begin{tabular}{|c|c|c|}
\hline$s_{i}$ & $p_{i}$ & $\ell_{i}$ \\
\hline \hline$a$ & .2 & 2 \\
\hline$b$ & .19 & 3 \\
\hline$c$ & .19 & 3 \\
\hline$d$ & .15 & 2 \\
\hline$e$ & .14 & 3 \\
\hline$f$ & .13 & 3 \\
\hline
\end{tabular}

Observe that $\ell_{i}=3>2=\ell_{j}$, where $i=3<4=j$. The corresponding locations in the list are $t_{9}$ and $t_{6}$ whose weights are 0.19 and 0.15 respectively. The sibling property is violated, since $0.19=p_{3}>p_{4}=0.15$.

Example 1 depicts a rather simple case, which is likely to occur when constructing the tree for real-life scenarios using Fano's method. We will observe this later when we present our empirical simulations on real-life files from standard benchmarks.

Theorem 1. Let $\mathcal{T}$ be a binary coding tree constructed from the source alphabet $\mathcal{S}=\left\{s_{1}, \ldots, s_{m}\right\}$ whose probabilities of occurrence $\mathcal{P}=\left[p_{1}, \ldots, p_{m}\right]$ are sorted, i.e. $p_{1} \geq \ldots \geq p_{m}$. For any encoding scheme, $\phi: \mathcal{S} \rightarrow\left\{w_{1}, \ldots, w_{m}\right\}$, obtained after labeling as per the tree $\mathcal{T}$, where $\ell_{i}$ is the length of $w_{i}$, if there exist $i$ and $j$ such that $i<j$ and $\ell_{i}>\ell_{j}$, then $\mathcal{T}$ does not satisfy the sibling property.

Sketch of Proof. Let $\mathcal{T}^{\star}=\left\{t_{1}^{\star}, \ldots, t_{2 m-1}^{\star}\right\}$ be a binary coding tree. $\mathcal{T}^{\star}$ satisfies the sibling property if and only if its nodes can be arranged in a sequence, $t_{1}^{\star}, t_{2}^{\star}, \ldots, t_{2 m-1}^{\star}$, such that all non-root nodes of the form $t_{2 i}^{\star}$ and $t_{2 i+1}^{\star}$ are siblings in $\mathcal{T}^{\star}, i=1, \ldots, m-1$, and $\tau_{2}^{\star} \geq \tau_{3}^{\star} \geq \ldots \geq \tau_{2 m-1}^{\star}$.

Let $\phi: s_{1} \rightarrow w_{1}, \ldots, s_{m} \rightarrow w_{m}$ be an encoding scheme generated from the coding tree $\mathcal{T}=\left\{t_{1}, \ldots, t_{2 m-1}\right\}$. Also, let $\ell_{i}$ is the length of $w_{i}$. Suppose that $\exists i$ and $j, i<j$, such that $\ell_{i}>\ell_{j}$. The proof is done by showing that the leaves associated with $s_{i}$ and $s_{j}, t_{k}$ and $t_{l}$, violate the sibling property for the three possible cases encountered:

(i) $t_{k}$ and $t_{l}$ are siblings.

(ii) $t_{k}$ and $t_{l}$ are not siblings, but they are in the same level.

(iii) $t_{k}$ and $t_{l}$ are not in the same level.

The details of the proof are omitted, and can be found in (Rueda, 2002; Rueda and Oommen, 2001).

The reader will observe from Theorem 1 that when there is a leaf whose weight is greater than that of a leaf located in a lower level of the tree, the sibling property is not satisfied. As a consequence, the average code word length of any encoding scheme obtained from this tree is not minimal.

\section{The Enhanced Coding Algorithm}

Considering the facts discussed above, we now propose $\mathrm{Fano}^{+}$using the following modification to the traditional static Fano's method. It is well known that Fano's method requires that the source symbols and their 
probabilities are sorted in a non-increasing order of the probabilities. What we incorporate is as follows: After

all the code words are generated, we sort them in terms of their increasing order of lengths maintaining $\mathcal{S}$ and $\mathcal{P}$ in the order of the probabilities. This enhancement leads to $\mathrm{Fano}^{+}$, a modified Fano coding algorithm which generates encoding schemes whose average code word lengths are arbitrarily close to those of the optimal ones (i.e. those generated by Huffman's algorithm). This enhancement is formalized in Rule 1 given below.

Rule 1. Consider the source alphabet $\mathcal{S}=\left\{s_{1}, \ldots, s_{m}\right\}$ whose probabilities of occurrence are $\mathcal{P}=\left[p_{1}, \ldots, p_{m}\right]$, where $p_{1} \geq p_{2} \geq \ldots \geq p_{m}$. Suppose that $\phi: s_{1} \rightarrow w_{1}, \ldots, s_{m} \rightarrow w_{m}$ is the encoding scheme obtained by Fano's method. Rearrange $w_{1}, \ldots, w_{m}$ into $w_{1}^{\prime}, \ldots, w_{m}^{\prime}$ such that $\ell_{i}^{\prime} \leq \ell_{j}^{\prime}$ for all $i<j$, and simultaneously maintain $s_{1}, \ldots, s_{m}$ in the same order, to yield the encoding scheme: $\phi^{\prime}: s_{1} \rightarrow w_{1}^{\prime}, \ldots, s_{m} \rightarrow w_{m}^{\prime}$.

The encoder construct a code, $\phi$, using the static Fano's method, obtains the enhanced encoding scheme $\phi^{\prime}$ by applying Rule 1 . The decoder invokes the same rule obtaining $\phi^{\prime}$, from which it generates the decoding scheme, $\left(\phi^{\prime}\right)^{-1}: w_{1}^{\prime} \rightarrow s_{1}, \ldots, w_{m}^{\prime} \rightarrow s_{m}$.

In Example 1, swapping the symbols $b$ and $c$ (and updating the content of the tree), is equivalent to swapping their corresponding code words in any of the encoding schemes generated from $\mathcal{T}$. Observe that after this modification, $\mathcal{T}$ satisfies the sibling property. Note that we do not obtain the optimal encoding algorithm, like Huffman's, since we are only swapping code words, and hence the coding tree is the same as that constructed by Fano's method. There are some cases in which the structure of the tree must be changed so that it satisfies the sibling property.

The procedure for generating the encoding scheme (which also includes the sorting process) is given in Algorithm Fano $^{+}$below.

The sorting procedure works, using the principles of radix sorting (Andersson et al., 1998), as follows. First, an array of $m-1$ elements is created, where the $i^{\text {th }}$ cell allocates the code words of length $\ell_{i}$. This is accomplished in the first for loop. In the second for loop, the entries of the array are scanned in canonical order, and for each entry (the inner for loop), the code words are "popped" from the array and allocated in the list of code words. As a result, the code words will be sorted in a non-decreasing order, and the probabilities of the symbols will preserve the original order, i.e. decreasing order.

Using the principles of radix sorting, an array of $m$ integers in the range $1 \ldots k, k=O(m)$, can be sorted in $O(m)$ time and space. Note that in the sorting procedure, the sum of sizeOf(temp $[i])$ is actually the number of code words, which is $m$, and hence the worst-case time complexity of procedure sortSymbols $(\ldots)$ is $O(m)$. In the actual implementation, a pointer to each code word (as opposed to the code word itself) could be stored in array "term", in order to achieve linear space complexity.

Observe that $\mathrm{Fano}^{+}$yields a coding sequence for every source alphabet symbol. The actual encoding and decoding are both therefore straightforward. Elementary coding textbooks report how such table-based prefix codes can be implemented efficiently.

\section{Properties of the Enhanced Fano Coding}

To facilitate the analysis, we first introduce two important properties of $\mathrm{Fano}^{+}$, the enhanced static Fano coding. The first relates to the efficiency in compression achieved by $\mathrm{Fano}^{+}$, and the second is the property 


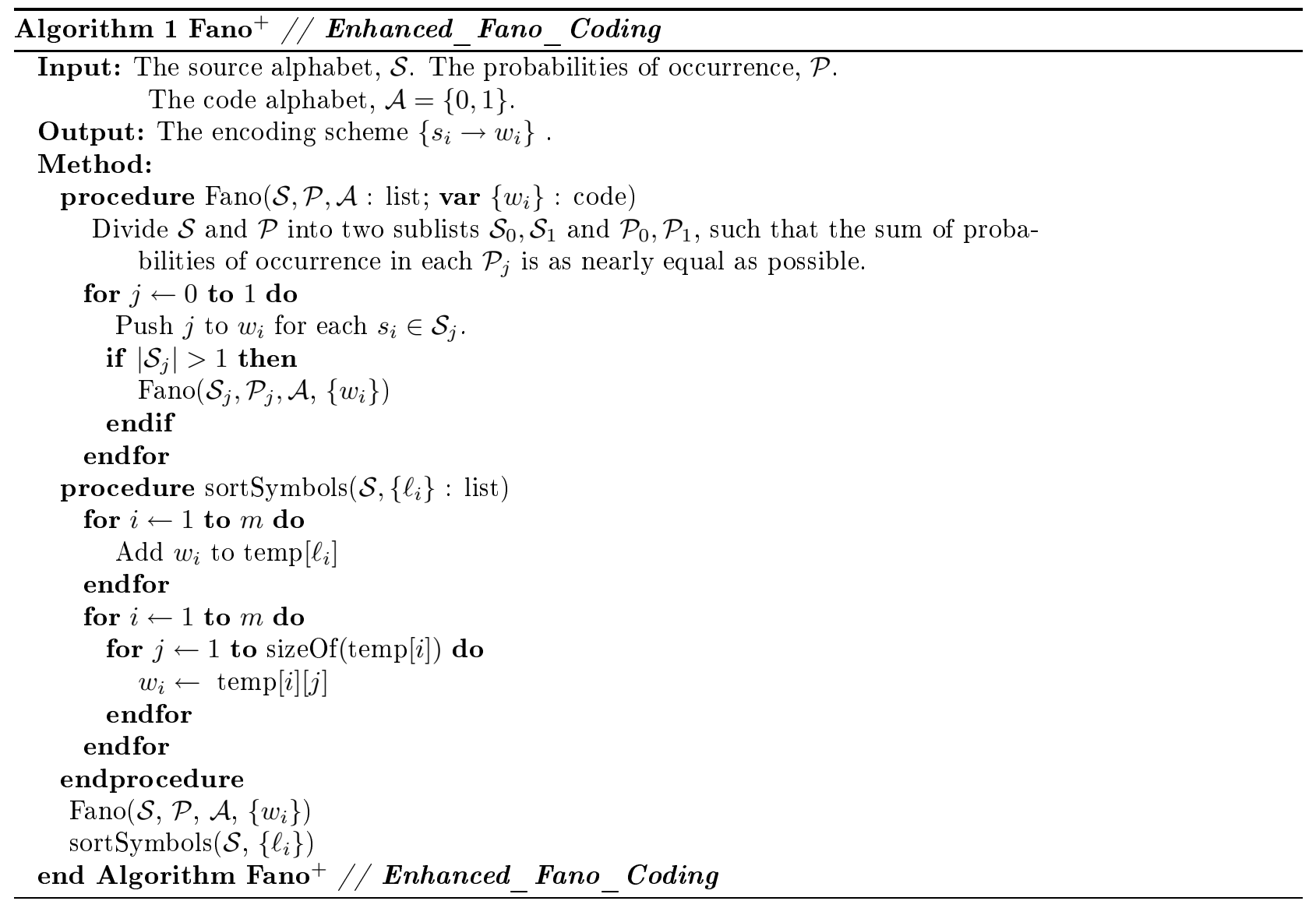

that it achieves lossless compression.

The efficiency of compression of $\mathrm{Fano}^{+}$is a direct consequence of the rearrangement of the code words such that they are sorted in an increasing order of length (Rule 1). This is stated in the following theorem, for which a proof can be found in (Hardy et al., 1959) ${ }^{2}$.

Theorem 2. Let $p_{1}, \ldots, p_{m}$ be positive real numbers such that $p_{1} \geq \ldots \geq p_{m}$, and let $\ell_{1}, \ldots, \ell_{m}$ be positive integers such that $\ell_{1} \leq \ldots \leq \ell_{m}$. Then, for any rearrangement $\ell_{1}^{\prime}, \ldots, \ell_{m}^{\prime}$ of the list $\ell_{1}, \ldots, \ell_{m}$,

$$
\sum_{i=1}^{m} p_{i} \ell_{i} \leq \sum_{i=1}^{m} p_{i} \ell_{i}^{\prime} .
$$

Observe that from Theorem 2, we can infer that for any list of code word lengths, we can obtain the optimal rearrangement, i.e. the one that satisfies $\ell_{1} \leq \ldots \leq \ell_{m}$, by using Rule 1 . However, this does not guarantee that we obtain the optimal encoding scheme (as in Huffman's algorithm), which also depends on constructing the optimal coding tree for a particular set of probabilities, $p_{1}, \ldots, p_{m}$.

The following counter-example shows that even the enhanced Fano coding algorithm does not ensure the optimal encoding scheme.

Example 2. Consider the source alphabet $\mathcal{S}=\{a, b, c, d, e\}$ whose probabilities of occurrence are $\mathcal{P}=$ $[.35, .17, .17, .16, .15]$. The coding tree constructed using the conventional Fano's method is depicted in Figure 2 (a). The corresponding Huffman tree is depicted in Figure 2 (b). Observe that even after rearranging the code words obtained from labeling the tree of Figure 2 (a), it is not possible to achieve the optimal encoding

\footnotetext{
${ }^{2}$ The proof given in (Hardy et al., 1959) is also valid for all real, not necessarily positive $p_{1}, \ldots, p_{m}$ and $\ell_{1}, \ldots, \ell_{m}$.
} 


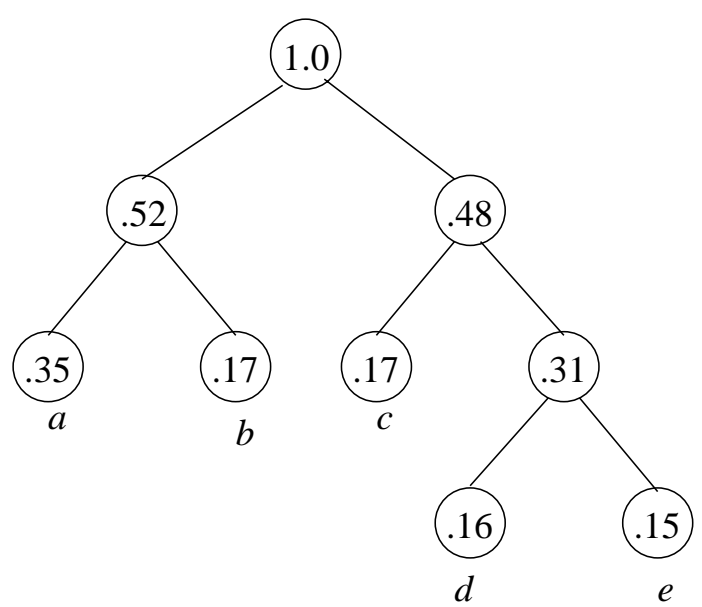

(a) Using Fano's method.

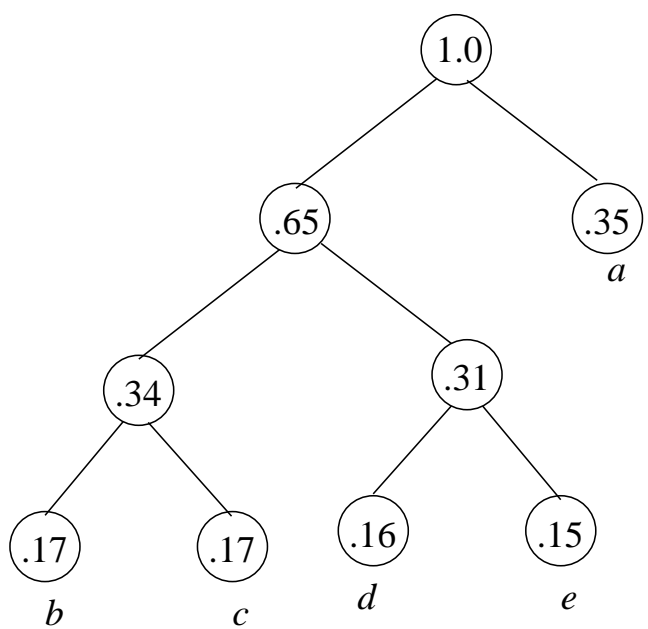

(b) Using Huffman's algorithm.

Figure 2: Two different binary coding trees constructed with the source alphabet and probabilities of occurrence given in Example 2.

scheme. This fact will also be observed in the empirical results shown later in this paper.

To initiate discussions on the analysis of $\mathrm{Fano}^{+}$, we first prove that it generates a prefix code. Note that this cannot be proven by using Kraft's inequality, since the latter is a necessary condition on the lengths of the code words, but not sufficient on the symbols composing the code words themselves.

Theorem 3. Let $\mathcal{S}=\left\{s_{1}, \ldots, s_{m}\right\}$ be the source alphabet, and $\mathcal{A}=\{0,1\}$ be the binary code alphabet. Procedure Fano(...) of Algorithm Fano ${ }^{+}$generates a prefix code.

Sketch of Proof. This theorem can be proven by an induction on the number of steps used in the partitioning. Let $\mathcal{S}(j)$ be the list being partitioned at time ' $j$ ', i.e. the $j^{\text {th }}$ partitioning step of the invoked procedure Fano(...). At the time instant ' $j$ ', $\mathcal{S}(j)$ is partitioned into $\mathcal{S}_{0}(j)$ and $\mathcal{S}_{1}(j)$. The symbol ' 0 ' is added as a suffix to all the code words of $\mathcal{S}_{0}(j)$, and ' 1 ' is added as a suffix to all the codewords of $\mathcal{S}_{1}(j)$.

The basis case is straightforward, as it involves the initial partitioning in which $\mathcal{S}(1)$ is partitioned into $\mathcal{S}_{0}(1)$ and $\mathcal{S}_{1}(1)$, where the code words of $\mathcal{S}_{0}(1)$ start with ' 0 ' and the code words of $\mathcal{S}_{1}(1)$ start with ' 1 '. Clearly, no code word of $\mathcal{S}_{0}(1)$ is a prefix of any code word in $\mathcal{S}_{1}(1)$ and vice versa.

The inductive step involves proving the hypothesis that no code word in $\mathcal{S}_{0}(j-1)$ is a prefix of any code word in $\mathcal{S}_{1}(j-1)$ and vice versa. This proof follows the exact same steps of the basis case in which we examine the symbols that occur after the $(j-1)^{s t}$. The details are actually not too complicated, although the symbolic narration is cumbersome. The complete proof can be found in (Rueda, 2002; Rueda and Oommen, 2001).

Theorem 4. Consider the source alphabet $\mathcal{S}=\left\{s_{1}, \ldots, s_{m}\right\}$ whose probabilities of occurrence are $\mathcal{P}=$ $\left[p_{1}, \ldots, p_{m}\right]$, where $p_{1} \geq \ldots \geq p_{m}$, and the binary code alphabet. Suppose that $\mathcal{X}$ is encoded into $\mathcal{Y}$ using Algorithm Fano $^{+}$. Then $\mathcal{X}$ is efficiently encoded into $\mathcal{Y}$, yielding an efficiency at least as that obtained using the conventional Fano's method.

Proof. The proof follows by a direct invocation of the properties of Theorems 1 and 2 . 


\begin{tabular}{|c|c|c|c|c|c|c|c|}
\hline \multirow{2}{*}{$\begin{array}{c}\text { File } \\
\text { Name }\end{array}$} & \multirow{2}{*}{$\begin{array}{l}\text { Orig. } \\
\text { Size: } l_{\mathcal{X}} \\
\text { (bytes) }\end{array}$} & \multicolumn{2}{|c|}{ Huffman } & \multicolumn{2}{|c|}{ Fano } & \multicolumn{2}{|c|}{ Fano $^{+}$} \\
\hline & & $\begin{array}{c}\ell y \\
\text { (bytes) }\end{array}$ & $\begin{array}{c}\rho \\
(\%)\end{array}$ & $\begin{array}{c}\ell y \\
\text { (bytes) }\end{array}$ & $\begin{array}{c}\rho \\
(\%)\end{array}$ & $\begin{array}{c}\ell \mathcal{Y} \\
\text { (bytes) }\end{array}$ & $\begin{array}{c}\rho \\
(\%)\end{array}$ \\
\hline bib & $\overline{111,261}$ & $\overline{73,003}$ & 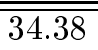 & $\overline{773,133}$ & 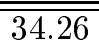 & $\overline{73,010}$ & "34.37 \\
\hline book1 & 768,771 & 438,619 & 42.94 & 439,322 & 42.85 & 438,803 & 42.92 \\
\hline book2 & 610,856 & 368,587 & 39.66 & 369,691 & 39.47 & 369,537 & 39.50 \\
\hline geo & 102,400 & 73,323 & 28.39 & 73,714 & 28.01 & 73,602 & 28.12 \\
\hline news & 377,109 & 246,687 & 34.58 & 246,890 & 34.53 & 246,773 & 34.56 \\
\hline obj1 & 21,504 & 16,819 & 21.78 & 16,845 & 21.66 & 16,822 & 21.77 \\
\hline obj2 & 246,814 & 194,863 & 21.04 & 195,324 & 20.86 & 194,933 & 21.02 \\
\hline paper1 & 53,161 & 33,621 & 36.75 & 33,655 & 36.69 & 33,653 & 36.69 \\
\hline progc & 39,611 & 26,189 & 33.88 & 26,355 & 33.46 & 26,326 & 33.53 \\
\hline progl & 71,646 & $\overline{43,242}$ & 39.64 & 43,588 & 39.16 & 43,529 & 39.24 \\
\hline progp & 49,379 & 30,480 & 38.27 & 30,527 & 38.17 & 30,501 & 38.23 \\
\hline trans & 93,695 & 65,514 & 30.08 & 65,723 & 29.85 & 65,515 & 30.08 \\
\hline Total & $2,547,207$ & $1,610,947$ & 36.76 & $1,614,767$ & 36.61 & $1,613,004$ & 36.68 \\
\hline
\end{tabular}

Table 1: Empirical results obtained after compressing test files from the Calgary corpus by using the static Huffman's algorithm, the static Fano's method, and the sibling-enhanced version of the static Fano's method $\left(\mathrm{Fano}^{+}\right)$.

\section{$5 \quad$ Empirical Results}

In order to analyze the efficiency of $\mathrm{Fano}^{+}$, we have conducted some experiments on files of the Calgary corpus ${ }^{3}$ and the Canterbury corpus (Witten et al., 1999). The empirical results obtained are displayed in Tables 1 and 2 respectively. The columns labeled 'Huffman' and 'Fano' correspond to the static Huffman's algorithm and the traditional static Fano's method respectively. The columns labeled 'Fano ${ }^{+}$' correspond to the siblingenhanced version of the static Fano's method introduced in this paper. The columns labeled ' $\ell$ ' represent the size (in bytes) of the compressed file. The columns labeled ' $\rho$ ' tabulate the percentage of compression obtained by the different methods, calculated as $\rho=\left(1-\frac{\ell_{\mathcal{Y}}}{l_{\mathcal{X}}}\right) 100$, where $l_{\mathcal{X}}$ is the length of the input file. The last row contains the total for each column except for the column labeled ' $\rho$ ', for which the value of the cell corresponds to the weighted average of compression ratio.

Observe that the gain in percentage of compression is $0.06 \%$ and $0.07 \%$ on the files of the Calgary corpus and the Canterbury corpus respectively. For example, for the file trans, Huffman and Fano ${ }^{+}$attain the same percentage of compression, e.g. 30.08\%, whereas the traditional Fano coding compresses slightly less, e.g. 29.85\%. Although the improved version of Fano's method does not guarantee the optimal encoding scheme, the weighted averages obtained from Fano ${ }^{+}$are significantly closer to those obtained by Huffman's algorithm. Observe also that in all the files, there is some gain in the compression ratio. This implies that in all the files encoded, $\ell_{1} \leq \ell_{2} \leq \ldots \leq \ell_{m}$ was not satisfied by the encoding schemes generated by the traditional static Fano's method, and validates the results for such an enhancement.

We also observe that the compression ratios achieved by our implementation of $\mathrm{Fano}^{+}$, which are very close to the optimal, can be significantly improved by incorporating higher-order structure models, such as dictionary based methods, Markovian models, etc. This is currently being investigated.

\footnotetext{
${ }^{3}$ Electronically available at ftp.cpsc.ucalgary.ca/pub/projects/text . compression. corpus/.
} 


\begin{tabular}{|c|c|c|c|c|c|c|c|}
\hline \multirow{2}{*}{$\begin{array}{c}\text { File } \\
\text { Name }\end{array}$} & \multirow{2}{*}{$\begin{array}{l}\text { Orig. } \\
\text { Size: } l_{\mathcal{X}} \\
\text { (bytes) }\end{array}$} & \multicolumn{2}{|c|}{ Huffman } & \multicolumn{2}{|c|}{ Fano } & \multicolumn{2}{|c|}{ Fano $^{+}$} \\
\hline & & $\begin{array}{c}\ell y \\
\text { (bytes) }\end{array}$ & $\begin{array}{c}\rho \\
(\%)\end{array}$ & $\begin{array}{c}\ell \mathcal{Y} \\
\text { (bytes) }\end{array}$ & $\begin{array}{c}\rho \\
(\%)\end{array}$ & $\begin{array}{c}\ell \mathcal{Y} \\
\text { (bytes) }\end{array}$ & $\begin{array}{c}\rho \\
(\%)\end{array}$ \\
\hline alice29.txt & $\overline{148,481}$ & 84,765 & 4242.91 & 85,254 & 42.58 & 84,999 & 42.75 \\
\hline asyoulik.txt & 125,179 & 76,010 & 39.27 & 76,195 & 39.13 & 76,124 & 39.18 \\
\hline cp.html & 24,603 & 16,456 & 33.11 & 16,477 & 33.02 & 16,458 & 33.10 \\
\hline fields.c & 11,150 & 7,295 & 34.56 & 7,354 & 34.03 & 7,349 & 34.08 \\
\hline grammar.lsp & 3,721 & 2,397 & 35.56 & 2,402 & 35.44 & 2,397 & 35.56 \\
\hline kennedy.xls & $1,029,744$ & 463,300 & 55.00 & 465,368 & 54.80 & 464,612 & 54.88 \\
\hline ice10.txt & 419,235 & 244,124 & 41.76 & 244,197 & 41.75 & 244,141 & 41.76 \\
\hline plrabn12.txt & 471,162 & 266,423 & 43.45 & 266,984 & 43.33 & 266,592 & 43.41 \\
\hline ptt5 & 513,216 & 107,027 & 79.14 & 107,138 & 79.12 & 107,075 & 79.13 \\
\hline sum & 38,240 & 26,409 & 30.93 & 26,501 & 30.69 & 26,463 & 30.79 \\
\hline xargs.1 & 4,227 & 2,823 & 33.20 & 2,825 & 33.16 & 2,824 & 33.18 \\
\hline Total & $2,788,958$ & $\begin{array}{l}1,297,029 \\
\end{array}$ & "53.49 & $1,300,695$ & "53.36 & $\overline{11,299,034}$ & $\overline{553.42}$ \\
\hline
\end{tabular}

Table 2: Details of the experiments performed with the static version of Huffman's algorithm, the traditional static Fano's method and the sibling-enhanced version of static Fano's method on files of the Canterbury corpus.

\section{Conclusions}

In this paper, we present an encoding scheme generation algorithm which is Fano-based and almost optimal. We first showed that for the encoding schemes, whose code words are not arranged in an increasing order of lengths, the corresponding coding tree does not satisfy the so-called sibling property. To rectify this, we introduced an enhanced version of the static Fano's method, Fano ${ }^{+}$, whose properties have been formally proven.

The encoding algorithm associated with $\mathrm{Fano}^{+}$have been formally presented, rigorously analyzed, and empirically tested. Our empirical results on files of the Calgary corpus and the Canterbury corpus show that $\mathrm{Fano}^{+}$achieves percentages of compression which are almost optimal - very marginally below those of Huffman's algorithm.

The extension of Fano ${ }^{+}$for multi-symbol code alphabets follows directly from the binary solution proposed. The main problem in dealing with multi-symbol code alphabets is that a more elaborate partitioning procedure is required, which we propose in (Rueda, 2002). The zeroth-order model implemented here can also be extended to higher order models such as dictionary-based methods, Markovian models, etc., so as to achieve compression ratios comparable to those of the best state-of-art compression methods.

\section{References}

Andersson, A., Hagerup, H., Nilsson, S., and Raman, R. (1998). Sorting in Linear Time? Journal of Computer and System Sciences, 57:74-93.

Faller, N. (1973). An Adaptive System for Data Compression. Seventh Asilomar Conference on Circuits, Systems, and Computers, pages 593-597.

Gallager, R. (1978). Variations on a Theme by Huffman. IEEE Transactions on Information Theory, 24(6):668674. 
Hankerson, D., Harris, G., and Jr., P. J. (1998). Introduction to Information Theory and Data Compression. CRC Press.

Hardy, G., Littlewood, J., and Póyla, G. (1959). Inequalities. Cambridge University Press, 2nd. edition.

Huffman, D. (1952). A Method for the Construction of Minimum Redundancy Codes. Proceedings of IRE, 40(9):1098-1101.

Kieffer, J. C. and Yang, E. (2000). Grammar-Bassed Codes: A new Class of Universal Lossless Source Codes. IEEE Transactions on Information Theory, 46(3):737-754.

Knuth, D. (1985). Dynamic Huffman Coding. Journal of Algorithms, 6:163-180.

Rueda, L. (2002). Advances in Data Compression and Pattern Recognition. PhD thesis, School of Computer Science, Carleton University, Ottawa, Canada.

Rueda, L. and Oommen, B. J. (2001). Nearly Optimal Fano-Based Canonical Codes. Technical Report SCS TR-01-05, School of Computer Science, Carleton University, Ottawa, Canada.

Sayood, K. (2000). Introduction to Data Compression. Morgan Kaufmann, 2nd. edition.

Shannon, C. E. and Weaver, W. (1949). The Mathematical Theory of Communications. University of Illinois Press.

Storer, J. (1988). Data Compression: Methods and Theory. Computer Science Press.

Witten, I., Moffat, A., and Bell, T. (1999). Managing Gigabytes: Compressing and Indexing Documents and Images. Morgan Kaufmann, 2nd. edition.

Ziv, J. and Lempel, A. (1977). A Universal Algorithm for Sequential Data Compression. IEEE Transactions on Information Theory, 23(3):337-343.

Ziv, J. and Lempel, A. (1978). Compression of Individual Sequences via Variable-Rate Coding. IEEE Transactions on Information Theory, 25(5):530-536. 